\title{
EDITORIAL
}

\section{Drug resistant tuberculosis: back to sanatoria, surgery and cod-liver oil?}

\author{
J. Veen
}

The decline of tuberculosis in industrialized countries and the emergence of effective antituberculosis drugs led to the optimistic belief that global eradication of tuberculosis could be achieved in a few decades. Preempting this eradication, politicians started to channel funds away from tuberculosis control. Already in 1953, Dubos and Dubos [1], warned: "The unit cost of detection of one new case of tuberculosis will probably increase as the prevalence of infection decreases and there is reason to fear that many communities will abandon too soon the search for active cases". Forty years later, REICHMAN [2] aptly described this ubiquitous phenomenon in public health practice as the "U-shaped curve of concern". When indicators show a decline, resources are removed, which is then followed by a rise in incidence that is proportionate to the diminished resources.

The decline of tuberculosis changed to an increase in the mid-eighties. This change was first noticed in the United States. The increasing incidence of tuberculosis was attributed to the human immunodeficiency virus (HIV) epidemic, poverty and the decline of tuberculosis control programmes [3]. Outbreaks and ominous reports of nosocomial transmission of multidrug resistant strains of Mycobacterium tuberculosis, also infecting hospital staff, rekindled interest in the "Captain of all the Men of Death", especially after RYAN [4] called the multidrugresistant strain a "superbug".

Multidrug-resistant tuberculosis has been described as the third epidemic, complicating the epidemics of acquired immune deficiency syndrome (AIDS) and tuberculosis, requiring urgent attention to achieve more rapid diagnosis, to develop new therapeutic regimens and to address the social and hospital environment to care for these individuals [5].

That resistance could emerge in antituberculous treatment was recognized early after the introduction of streptomycin. Monotherapy with this drug led to $80 \%$ resistance within 3 months of commencement of therapy [6], while monotherapy with isoniazid caused resistance in $30 \%$ of patients [7]. To prevent and treat drug-resistant tuberculosis one has to understand how resistance can occur. Drug resistance of M. tuberculosis develops by the selective growth of resistant mutants evolving in a wild population, when exposed to a sufficient amount of drugs that can inhibit growth of sensitive

Correspondence: J. Veen, Royal Netherlands Tuberculosis Association, P.O. Box 146, 2501 CC The Hague, The Netherlands. organisms [8]. YEW and CHAU [9] in a review in the Journal explain that the incidence of drug resistant mutants is related to the absolute number of bacilli in the lesion and the probability of becoming a resistant mutant against a particular drug. The probability of spontaneous mutation to two or more drugs is the product of the individual probabilities and becomes very low. This mutation is a natural process, and as such it cannot be influenced by man. Fortunately, the chromosomal loci for resistance to the various drugs are not linked.

The presence of a few resistant mutants does not cause drug-resistant tuberculosis when the treatment regimen is adequate. It is inadequate treatment that, through selection of resistant mutants, results in secondary (or acquired) resistance. Transmission of resistant strains may occcur and contacts develop resistant primary tuberculosis [10].

In discussing drug resistance, it is important to be very clear about definitions. YEW and $\mathrm{CHAU}$ [9] define acquired resistance, which is a better term than secondary resistance, since it describes the mechanism. Primary resistance can be used if one is absolutely certain that no prior treatment took place, but has to be replaced by initial resistance in most instances. This is more than just an academic difference. Acquired resistance arises during the course of treatment. Therefore, a high level of this type of resistance is a mark for a poorly functioning tuberculosis control programme in the current time, whilst primary resistance is present in a patient, who never received treatment, therefore must have been infected by a drug-resistant source, which is an indicator of the efficacy of tuberculosis control efforts in the past [8]. This relationship between treatment efficacy and resistance prevalence was shown very clearly in Korea. The prevalence of drug resistance was $48 \%$ in 1980 and fell to $2 \%$ in 1990 through improved programme efficacy due to the reduction of failure cases. Initial resistance decreased at a lower rate from $26 \%$ in 1965 to $15 \%$ in 1990 [11].

There is little knowledge about the global burden of (multi)drug-resistant tuberculosis. Some countries with a high incidence of disease seem to have a high rate of resistance: China 20\%, India 20\%, Pakistan $>30 \%$, Philippines $33 \%$ [12]. An accurate count of drug-resistant tuberculosis throughout the world is not available, because few countries (both industrialized and developing) have reliable drug resistance surveillance systems [13]. What is needed is standardized data-management, in which the key indicators as primary, initial, and acquired resistance are monitored and reported, both as absolute numbers 
and proportions. Uniform definitions for single drug and multidrug resistance should be used, and laboratory methods should be standardized.

Lack of standardization is illustrated by two articles about drug resistance in northern German hospitals [14, 15]. Both articles are similar in method of investigation, laboratory and statistical methods, number of patient records examined and results, including associated factors obtained; however, it is difficult to compare some of the basic data.

The prevalence of drug resistance is $9.7 \%$ and $9.6 \%$, the prevalence of single drug resistance in both studies is $5.9 \%$ and $5.8 \%$. But it needs careful reading and calculation to arrive at various other parameters. One article uses multidrug resistance as a synonym for resistance against more than one drug, whilst the other confines this term to resistance to isoniazid and rifampicin. Although combined resistance to isoniazid and rifampicin is reported, the Berlin study requires calculation to arrive at $1.2 \%$, whilst the Hamburg study reports $1.8 \%$. The latter reports no increasing trend, whilst the former reports a considerable increase in multidrug-resistant tuberculosis, but then uses the wider definition. It has become common practice to restrict multidrug resistance to at least the combined resistance to isoniazid and rifampicin. SCHABERG et al. [14] reason correctly that resistance to at least two first-line drugs will cause severe treatment problems, but uniformity in reporting increases understanding of programme and patient management. One would either need to include the drug's acronyms, such as HR-resistance (isoniazid/rifampicin) or use a term like polyresistance for resistance to more than one drug, keeping multidrug resistance exclusively for the combined resistance to isoniazid and rifampicin.

The similar findings in both hospitals do not necessarily reflect the situation in the whole of Germany. Leaving alone the fact that the prevalence of tuberculosis differed in former East Germany from West Germany, being a hospital study, there will be a selection bias towards the more difficult patients. The absolute numbers will probably reflect all resistant cases in the catchment area, the proportion will be an overestimate.

Retrospective studies tend to underestimate factors such as prior treatment, and some cases classified as acquired resistance may, in fact, have been initial resistance. Both studies are unanimous, however, in their findings of associated factors. Not surprisingly, prior treatment and being foreign born are significant contributors to the resistance problem.

These factors reflect the most important underlying cause for the development of resistance: noncompliance, being either noncompliance with programme policy or noncompliance in patient management. Compliance with programme policy requires the active involvement of politicians and public health policy makers. It might include provision of funds to support an effective structure, or to invest in health education of both the professionals and the general public. But it also means compliance of the provider with the use and delivery of adequate regimens and compliance of the patient with the treatment prescribed.
Provided adequate funds and professional skills are available for tuberculosis control, what measures can be taken to prevent the emergence and spread of resistance? In all cases where prior treatment cannot be excluded, initial treatment should contain a four drug regimen, containing at least two drugs with which the patient has not hitherto been treated. Patients from nonindustrialized countries in particular are at risk, although drug resistance remains low in extremely poor nations where the medications are scarce. Resistance rates are higher in economically intermediate countries, where there is sufficient wealth to purchase tuberculosis medications, but inadequate resources to organize or supervise therapy [12]. The use of combination tablets and strict supervision may prohibit the development of resistance, as shown by the experience in Tanzania [16].

For surveillance's sake, but also for monitoring the progress of the individual patient, susceptibility testing in all first isolates needs to be carried out, if funds permit. Otherwise, all first isolates of relapse cases (did have prior treatment) or isolates of failure cases (no improvement during therapy) should be tested. Care should be taken not to supplement the regimens of nonresponding patients by adding individual drugs, since this will lead to a stepwise emergence of polyresistance (addition syndrome).

The selling of antituberculosis drugs should be legally controlled [17]. Compliance of the patient with the treatment regimen might be the most difficult aspect of the therapy. Not only is the regimen complicated through the use of multiple drugs, it also takes many months to complete. Regular supervision is needed, in which close surveillance by the health visitor is more effective than follow-up by the physician [18]. For patients who are difficult to manage, direct observed therapy (DOT) might be needed. One recent study showed that acquired resistance in unsupervised groups was as high as $14 \%$, whilst in patients managed with DOT it was only $2.1 \%$. DOT also proved to be a cost-effective intervention [19].

What about the contribution of the HIV epidemic? There is no reason to believe that treatment in HIVinfected tuberculosis patients is less effective as in nonHIV infected persons, although one might speculate that the ultimate clearance of the bacillus by the host requires an intact immune system. But the increased breakdown from infection to active disease from $10 \%$ in a lifetime to $10 \%$ annually in HIV-infected patients, will increase the caseload. Increased caseloads might lead to inadequate patient management, thereby causing more acquired resistance, followed by increased transmission of resistant strains and, thus, increase of primary resistance. HIV infection, therefore, may contribute indirectly to the emergence of resistance [10].

In conclusion, one may say that the global size of drugresistant tuberculosis is not known, since very few countries monitor resistance on a regular basis. The World Health Organization (WHO) wants to stimulate resistance surveillance. For that, uniform definitions and standardized procedures, including data-management are needed.

The key factor in the prevention of resistance is compliance of all parties involved in tuberculosis control. 
This is as much the responsibility of the patient and the professional, as the politician. As Dubos and Dubos [1] cite Machiavelli: "Consumption in the commencement is easy to cure, and difficult to understand; but when it has neither been discovered in due time, nor treated upon a proper principle, it becomes easy to understand, and difficult to cure. The same thing happens in state affairs, by foreseeing them at a distance, which is only done by men of talents, the evils which may arise from them are soon cured; but when, from want of foresight, they are suffered to increase to such a height that they are perceptible to everyone, there is no longer any remedy."

Professionals have diagnosed an emerging problem; both professionals and policy makers have to find a remedy. Otherwise, it is back to sanatoria, surgery and codliver oil.

\section{References}

1. Dubos R, Dubos J. The white plague. London, Gollancz Ltd, 1953.

2. Reichman LB. The U-shaped curve of concern. Am Rev Respir Dis 1991; 144: 741-742.

3. Bloom BR, Murray CJL. Tuberculosis: commentary on a re-emergent killer. Science 1992; 257: 1055-1064.

4. Ryan F. Tuberculosis: the greatest story never told. England, Swift Publishers, 1992.

5. Neville K, Bromberg A, Bromberg R, et al. The third epidemic: multidrug-resistant tuberculosis. Chest 1994; 105: 45-48.

6. Medical Research Council Investigations. Streptomycin treatment of pulmonary tuberculosis. Br Med J 1948; 2: 769-782.

7. Selkon JB, Devadatta S, Kulkarni KG, et al. The emergence of isoniazid-resistant cultures in patients with pulmonary tuberculosis during treatment with isoniazid alone or isoniazid plus PAS. Bull WHO 1964; 31: 273294.

8. Kochi A, Vareldzis B, Styblo K. Multidrug resistant tuberculosis and its control. Res Microbiol 1993; 144: 104-110.

9. Yew WW, Chau CH. Drug-Resistant Tuberculosis in the 1990s. Eur Respir J 1995; 8: 1184-1192.

10. Lambregts-van Weezenbeek CSB, Veen J. Control of drug resistant tuberculosis. Tuberc Lung Dis Vol. 76, (in press).

11. Kim SJ, Hong YP. Drug resistance of Mycobacterium tuberculosis in Korea. Tuberc Lung Dis 1992; 73: 219_ 224.

12. Iseman MD, Madsen LA. Drug resistant tuberculosis. Clin Chest Med 1989; 10: 341-353.

13. Raviglione MC, Snider DE Jr, Kochi A. Global epidemiology of tuberculosis. J Am Med Assoc 1995; 273 : 220-226.

14. Schaberg T, Gloger G, Reichert B, et al. Drug-resistant pulmonary tuberculosis in Berlin, Germany 1987-1993. Eur Respir J 1995; 8: 278-284.

15. Borchardt J, Kirsten D, Joerres R, et al. Drug resistant tuberculosis in Northern Germany: a retrospective hospital-based study of 1,055 patients from 1984 through 1993. Eur Respir J 1995; 8: 1076-1083.

16. Enarson DA. Principles of IUATLD collaborative tuberculosis programmes. Bull IUATLD 1991; 66: 195200.

17. Crofton J. Tuberculosis: propter Koch, post Koch: a global review. IUATLD Newsletter 1994; Dec: 2-7.

18. Ormerod LP, Prescott RJ. Interrelations between relapses, drug regimens and compliance with treatment in tuberculosis. Respir Med 1991; 85: 239-242.

19. Weis SE, Slocum PC, Blais FX, et al. The effect of directly observed therapy on the rates of drug resistance and relapse in tuberculosis. N Engl J Med 1994; 30: 1179-1184. 